\title{
The Bäcklund Transformations, Exact Solutions, and Conservation Laws for the Compound Modified Korteweg-de Vries-Sine-Gordon Equations which Describe Pseudospherical Surfaces
}

\author{
S. M. Sayed ${ }^{1,2}$ \\ ${ }^{1}$ Mathematics Department, Faculty of Science, Tabouk University, Tabouk, Saudi Arabia \\ ${ }^{2}$ Mathematics Department, Faculty of Science, Beni-Suef University, Beni-Suef, Egypt \\ Correspondence should be addressed to S. M. Sayed; s_m_sayed71@yahoo.com
}

Received 14 November 2012; Revised 21 January 2013; Accepted 24 January 2013

Academic Editor: Laurent Gosse

Copyright (C) 2013 S. M. Sayed. This is an open access article distributed under the Creative Commons Attribution License, which permits unrestricted use, distribution, and reproduction in any medium, provided the original work is properly cited.

I show that the compound modified Korteweg-de Vries-Sine-Gordon equations describe pseudospherical surfaces, that is, these equations are the integrability conditions for the structural equations of such surfaces. I obtain the self-Bäcklund transformations for these equations by a geometrical method and apply the Bäcklund transformations to these solutions and generate new traveling wave solutions. Conservation laws for the latter ones are obtained using a geometrical property of these pseudospherical surfaces.

\section{Introduction}

A differential equation (DE) for a real-valued function $u(x, t)$ is said to describe pseudospherical surfaces (pss) if it is the necessary and sufficient condition for the existence of smooth functions $f_{i j}, 1 \leq i \leq 3,1 \leq j \leq 2$, depending on $u$ and its derivatives, such that the 1 -forms $\omega_{i}=f_{i 1} d x+f_{i 2} d t, 1 \leq$ $i \leq 3$ satisfy the structure equations of a surface of a constant Gaussian curvature equal to -1 , that is,

$$
d \omega_{1}=\omega_{3} \wedge \omega_{2}, \quad d \omega_{2}=\omega_{1} \wedge \omega_{3}, \quad d \omega_{3}=\omega_{2} \wedge \omega_{1} .
$$

It is equivalent to say that the $\mathrm{DE}$ for $u(x, t)$ is necessary and sufficient for the integrability of the linear system [1-6]

$$
d \phi=\Omega \phi, \quad \phi=\left(\begin{array}{l}
\phi_{1} \\
\phi_{2}
\end{array}\right)
$$

where $d$ denotes exterior differentiation, $\phi$ is a column vector, and the $2 \times 2$ matrix $\Omega\left(\Omega_{i j}, i, j=1,2\right)$ is traceless

$$
\Omega=\frac{1}{2}\left(\begin{array}{cc}
\omega_{2} & \omega_{1}-\omega_{3} \\
\omega_{1}+\omega_{3} & -\omega_{2}
\end{array}\right)
$$

Take

$$
\begin{aligned}
\Omega & =\left(\begin{array}{cc}
\frac{\eta}{2} d x+A d t & q d x+B d t \\
r d x+C d t & -\frac{\eta}{2} d x-A d t
\end{array}\right) \\
& =P d x+Q d t,
\end{aligned}
$$

from (2) and (4), we obtain

$$
\phi_{x}=P \phi, \quad \phi_{t}=Q \phi,
$$

where $P$ and $Q$ are two $2 \times 2$ null-trace matrices

$$
P=\left(\begin{array}{cc}
\frac{\eta}{2} & q \\
r & -\frac{\eta}{2}
\end{array}\right), \quad Q=\left(\begin{array}{cc}
A & B \\
C & -A
\end{array}\right) .
$$

Here, $\eta$ is a parameter, independent of $x$ and $t$, while $q$ and $r$ are functions of $x$ and $t$. Now,

$$
0=d^{2} \phi=d \Omega \phi-\Omega \wedge d \phi=(d \Omega-\Omega \wedge \Omega) \phi,
$$


which requires the vanishing of the two forms:

$$
\Theta \equiv d \Omega-\Omega \wedge \Omega=0
$$

or in the component form:

$$
\begin{gathered}
A_{x}=q C-r B, \\
q_{t}-2 A q-B_{x}+\eta B=0, \\
C_{x}=r_{t}+2 A r-\eta C .
\end{gathered}
$$

Many partial differential equations (PDEs) which are of interest to study and investigate due to the role they play in various areas of mathematics and physics are included in this category [7-25].

The formulation of classical theory of surfaces in a form is familiar to the soliton theory, which makes possible an application of the analytical methods of this theory to integrable cases [26].

The results of [27] were obtained by inverse spectral method. The results of [28] were obtained using algorithm for constructing certain exact solutions, such as solutions describing the interaction of two traveling waves.

The Fokas transform method for solving boundary value problems for linear and integrable nonlinear PDEs can be viewed as an extension of the Fourier transform method, and, indeed, how in simple cases it reduces to the Fourier transform. The unifying character of the steps involved in the Fokas method makes it attractive from the theoretical and formal point of view. For nonlinear integrable problems, this approach is, at present, the only existing method yielding results in a general context [29-32].

The Fokas method has a much broader domain of applicability than it is possible to present in [30-33]. For example, elliptic problems can also be treated by this general approach. In this case, the analysis of the global relation, which is the crucial step in the methodology, may involve the solution of additional Reimann-Hilbert problems. As regards numerical approximations, preliminary results indicate that, using this approach, the Dirichlet-to-Neumann map for linear elliptic problems can also be evaluated, at least for some important examples, with exponential accuracy $[34,35]$.

The current paper directions include the implementation of the geometrical properties and the Bäcklund transformations (BTs) to generate a new soliton solution and conservation laws for the compound modified Korteweg-de Vriessine-Gordon (cmKdV-SG) equations.

The paper is organized as follows. In Section 2, I show that the cmKdV-SG equations describe pss. In Section 3, we find the BTs for the cmKdV-SG equations. Exact soliton solution class from a known constant solution is obtained for the cmKdV-SG equations. On the other hand, a new exact traveling wave solutions for the cmKdV-SG equations are obtained by using the BTs to generate a new soliton solution class in Section 4. In Section 5, I obtain an infinite number of conserved densities for the cmKdV-SG equations which describe pss using a theorem of Khater et al. [18] and Sayed et al. [23-25]. Finally, I give some conclusions in Section 6.

\section{The cmKdV-SG Equations which Describe pss}

The notion of a DE describing pss was first introduced by Chern and Tenenblat [2], who observed that most of the nonlinear evolution equations (NLEEs) solvable by the method of inverse scattering [3-5], such as the $\mathrm{KdV}$ and $\mathrm{mKdV}$ equations, have the property of describing pss. They also showed that if $f_{21}=\eta$ and the functions $f_{11}$ and $f_{31}$ do not depend on $\eta$, then the linear system (2) reduces to the inverse scattering problem (ISP) considered by Ablowitz et al. in [3], with $\eta$ corresponding to the spectral parameter. Let $M^{2}$ be a two-dimensional differentiable manifold parameterized by coordinates $x, t$. We consider a metric on $M^{2}$ defined by $\omega_{1}, \omega_{2}$. The first two equations in (1) are the structure equations which determine the connection from $\omega_{3}$, and the last equation in (1), the Gauss equation, determines that the Gaussian curvature of $M^{2}$ is -1 , that is, $M^{2}$ is a pss. Moreover, the one-forms

$$
\begin{gathered}
\omega_{1}=f_{11} d x+f_{12} d t, \quad \omega_{2}=f_{21} d x+f_{22} d t \\
\omega_{3}=f_{31} d x+f_{32} d t
\end{gathered}
$$

satisfy the structure equations (1) of a pss. It has been known, for a long time, that the SG equation describes a pss. In this paper, we extend the same analysis to include the $\mathrm{cmKdV}-\mathrm{SG}$ equations:

$$
\beta u_{r \theta}+\frac{1}{16} h^{4}\left(u_{r}\right)^{2} u_{r r}+\frac{1}{24} h^{4} u_{r r r}-\alpha \sin u=0
$$

where $\alpha, \beta, h$ are constants. This equation can be thought of as a generalization of the $\mathrm{mKdV}$ and SG equations. As particular cases:

(i) when $\alpha=0$, (11) becomes the $\mathrm{mKdV}$ equation in $u_{r}$ which is retrieved,

(ii) while the neglect of the terms in $h^{4}$ leads to the SG equation. Moreover, the introduction of the variables

$$
x=(24)^{1 / 4} \frac{r}{h}, \quad t=(24)^{-1 / 4} \frac{h \theta}{\beta},
$$

reduces (11) to the form

$$
u_{x t}+u_{x x x x}+\frac{3}{2} u_{x}^{2} u_{x x}-\alpha \sin u=0 .
$$

Let $M^{2}$ be a differentiable surface, parameterized by coordinates $x, t$. Consider that

$$
\begin{gathered}
\omega_{1}=\left(\eta u_{x x}+\frac{\alpha}{\eta} \sin u\right) d t \\
\omega_{2}=\eta d x+\left(\frac{\alpha}{\eta} \cos u-\eta^{3}-\frac{\eta}{2} u_{x}^{2}\right) d t \\
\omega_{3}=u_{x} d x+\left(-u_{x x x}-\eta^{2} u_{x}-\frac{1}{2} u_{x}^{3}\right) d t,
\end{gathered}
$$

then $M^{2}$ is a pss if and only if $u$ satisfies the cmKdV-SG equations (13). 


\section{The Self-Bäcklund Transformation for the cmKdV-SG Equations}

In this section, we show how the geometrical properties of a pss may be applied to obtain analytical results for the $\mathrm{cmKdV}$ SG equations which describe pss.

The classical Bäcklund theorem originated in the study of pss, relating solutions of the SG equation. Other transformations have been found relating solutions of specific equations in [6-9]. Such transformations are called BTs after the classical one. A BT which relates solutions of the same equation is called a self-Bäcklund transformation (sBT). An interesting fact which has been observed is that DEs which have sBT also admit a superposition formula. The importance of such formulas is due to the following: if $u_{0}$ is a solution of the NLEE and $u_{1}, u_{2}$ are solutions of the same equation obtained by the $\mathrm{SBT}$, then the superposition formula provides a new solution $u^{\prime}$ algebraically. By this procedure, one obtains the soliton solutions of an NLEE. In what follows, we show that geometrical properties of pss provide a systematic method to obtain the BTs for some NLEEs which describe pss.

Proposition 1. Given a coframe $\left\{\bar{\omega}_{1}, \bar{\omega}_{2}\right\}$ and corresponding connection one-form $\bar{\omega}_{3}$ on a smooth Riemannian surfaces $M^{2}$, there exists a new coframe $\left\{\bar{\omega}_{1}^{\prime}, \bar{\omega}_{2}^{\prime}\right\}$ and new connection oneform $\bar{\omega}_{3}^{\prime}$ satisfying the equations

$$
d \bar{\omega}_{1}^{\prime}=0, \quad d \bar{\omega}_{2}^{\prime}=\bar{\omega}_{2}^{\prime} \wedge \bar{\omega}_{1}^{\prime}, \quad \bar{\omega}_{3}^{\prime}+\bar{\omega}_{2}^{\prime}=0,
$$

if and only if the surface $M^{2}$ is pss. For the sake of clarity, one gives a revised proof of [10].

Proof. Assume that the orthonormal dual to the coframes $\left\{\bar{\omega}_{1}, \bar{\omega}_{2}\right\}$ and $\left\{\bar{\omega}_{1}^{\prime}, \bar{\omega}_{2}^{\prime}\right\}$ possess the same orientation. The oneforms $\bar{\omega}_{i}$ and $\bar{\omega}_{i}^{\prime} \quad(i=1,2,3)$ are connected by means of

$$
\begin{gathered}
\bar{\omega}_{1}^{\prime}=\bar{\omega}_{1} \cos \psi-\bar{\omega}_{2} \sin \psi, \quad \bar{\omega}_{2}^{\prime}=\bar{\omega}_{1} \sin \psi+\bar{\omega}_{2} \cos \psi, \\
\bar{\omega}_{3}^{\prime}=\bar{\omega}_{3}-d \psi .
\end{gathered}
$$

It follows that $\bar{\omega}_{1}^{\prime}, \bar{\omega}_{2}^{\prime}, \bar{\omega}_{3}^{\prime}$ satisfying (15) exist if and only if the Pfaffian system

$$
\bar{\omega}_{3}-d \psi+\bar{\omega}_{1} \sin \psi+\bar{\omega}_{2} \cos \psi=0,
$$

on the space of coordinates $(x, t, \psi)$ is completely integrable for $\psi(x, t)$, and this happens if and only if $M^{2}$ is pss.

Geometrically, (15) and (17) determine geodesic coordinates on $M^{2}$. Now, if $u_{t}=F\left(u, u_{x}, \ldots, u_{x^{k}}\right)\left(u_{x^{k}}=\partial^{k} u / \partial x^{k}\right)$ describes pss with associated one-forms $\omega_{i}=f_{i 1} d x+f_{i 2} d t$, (15) and (17) imply that the Pfaffian system,

$$
\omega_{3}-d \psi+\omega_{1} \sin \psi+\omega_{2} \cos \psi=0,
$$

is completely integrable for $\psi(x, t)$ whenever $u(x, t)$ is a local solution of $u_{t}=F\left(u, u_{x}, \ldots, u_{x^{k}}\right)[2,11]$.

Proposition 2. Let $u_{t}=F\left(u, u_{x}, \ldots, u_{x^{k}}\right)$ be an NLEE which describes a pss with associated one-forms (10). Then, for each solution $u(x, t)$ of $u_{t}=F\left(u, u_{x}, \ldots, u_{x^{k}}\right)$, the system of equations for $\psi(x, t)$,

$$
\begin{gathered}
\psi_{x}-f_{31}+f_{11} \sin \psi+\eta \cos \psi=0, \\
\psi_{t}-f_{32}+f_{12} \sin \psi+f_{22} \cos \psi=0,
\end{gathered}
$$

is completely integrable. Moreover, for each solution of $u(x, t)$ of $u_{t}=F\left(u, u_{x}, \ldots, u_{x^{k}}\right)$ and corresponding solution $\psi$,

$$
\left(f_{11} \cos \psi-\eta \sin \psi\right) d x+\left(f_{12} \cos \psi-f_{22} \sin \psi\right) d t
$$

is a closed one-form [2].

Eliminating $\psi(x, t)$ from (19), by using the substitution

$$
\cos \psi=\frac{2 \Gamma}{1+\Gamma^{2}}
$$

where

$$
\Gamma=\frac{\phi_{1}}{\phi_{2}},
$$

then (19) is reduced to the Riccati equations:

$$
\begin{array}{r}
\frac{\partial \Gamma}{\partial x}=\eta \Gamma+\frac{1}{2} f_{11}\left(1-\Gamma^{2}\right)-\frac{1}{2} f_{31}\left(1+\Gamma^{2}\right), \\
\frac{\partial \Gamma}{\partial t}=f_{22} \Gamma+\frac{1}{2} f_{12}\left(1-\Gamma^{2}\right)-\frac{1}{2} f_{32}\left(1+\Gamma^{2}\right) .
\end{array}
$$

The procedure in the following is that one constructs a transformation $\Gamma^{\prime}$ satisfying the same equation as (24) with a potential $u^{\prime}(x)$, where

$$
u^{\prime}(x)=u(x)+f(\Gamma, \eta) .
$$

Thus eliminating $\Gamma$ in (23), (24), and (25), we have a BT to a desired NLEE. We consider the following example (BT for the cmKdV-SG equations).

For (13), we consider the functions defined by

$$
\begin{gathered}
f_{11}=0, \quad f_{12}=\eta u_{x x}+\frac{\alpha}{\eta} \sin u, \\
f_{21}=\eta, \quad f_{22}=\frac{\alpha}{\eta} \cos u-\eta^{3}-\frac{\eta}{2} u_{x}^{2}, \\
f_{31}=u_{x}, \quad f_{32}=-u_{3 x}-\eta^{2} u_{x}-\frac{1}{2} u_{x}^{3},
\end{gathered}
$$

for any solution $u(x, t)$ of (13), the above functions satisfy (8). Then, (23) becomes

$$
\frac{\partial \Gamma}{\partial x}=\eta \Gamma-\frac{u_{x}}{2}\left(1+\Gamma^{2}\right) .
$$

If we choose $\Gamma^{\prime}$ and $u^{\prime}$ as

$$
\begin{gathered}
\Gamma^{\prime}=\frac{1}{\Gamma}, \\
u^{\prime}=u+4 \tan ^{-1} \Gamma,
\end{gathered}
$$


then $\Gamma^{\prime}$ and $u^{\prime}$ satisfy (27). If we eliminate $\Gamma$ in (27) and (24) with (28), we get the BT

$$
\begin{gathered}
\left(u^{\prime}+u\right)_{x}=-2 \eta \sin \frac{1}{2}\left(u-u^{\prime}\right), \\
\left(u-u^{\prime}\right)_{t}=2 f_{32}-2 f_{12} \cos \frac{1}{2}\left(u-u^{\prime}\right)+2 f_{22} \sin \frac{1}{2}\left(u-u^{\prime}\right) .
\end{gathered}
$$

Equation (29) is the BT for the cmKdV-SG equations (13) with $f_{12}, f_{22}$, and $f_{32}$ given in (26).

\section{A New Traveling Wave Solutions for the cmKdV-SG Equations}

For any solution $u(x, t)$ of the cmKdV-SG equations (13), the matrices $P$ and $Q$ are

$$
Q=\left(\begin{array}{cc}
P=\left(\begin{array}{cc}
\frac{\eta}{2} & -\frac{u_{x}}{2} \\
\frac{u_{x}}{2} & -\frac{\eta}{2}
\end{array}\right), \\
\frac{1}{2}\left(-\eta^{3}-\frac{\eta u_{x}^{2}}{2}+\frac{\alpha}{\eta} \cos u\right) & \frac{1}{2}\left(\eta u_{x x}+u_{x x x}+\frac{u_{x}^{3}}{2}+\eta^{2} u_{x}+\frac{\alpha}{\eta} \sin u\right) \\
\frac{1}{2}\left(\eta u_{x x}-u_{x x x}-\frac{u_{x}^{3}}{2}-\eta^{2} u_{x}+\frac{\alpha}{\eta} \sin u\right) & -\frac{1}{2}\left(-\eta^{3}-\frac{\eta u_{x}^{2}}{2}+\frac{\alpha}{\eta} \cos u\right)
\end{array}\right) .
$$

Substitute $u=n \Pi, n=0, \pm 1, \pm 2, \pm 3, \ldots$ into the matrices $P$ and $Q$ in (30), then by (5) we have

$$
d \phi=\phi_{x} d x+\phi_{t} d t=P \phi d \rho_{n}
$$

where

$$
\begin{gathered}
P=\left(\begin{array}{cc}
\frac{\eta}{2} & 0 \\
0 & -\frac{\eta}{2}
\end{array}\right), \\
\rho_{n}=x-k t, \quad k=\eta^{2}-\frac{\alpha}{\eta^{2}}(-1)^{n} .
\end{gathered}
$$

The solution of (31) is

$$
\phi_{n}=e^{\rho_{n} P} \phi_{0}=\left(I+\rho_{n} P+\frac{\rho_{n}^{2} P^{2}}{2 !}+\frac{\rho_{n}^{3} P^{3}}{3 !}+\cdots\right) \phi_{0},
$$

where $\phi_{0}$ is a constant column vector. The solution of (33) is

$$
\phi_{n}=\left(\begin{array}{cc}
\cosh \frac{\eta}{2} \rho_{n}+\sinh \frac{\eta}{2} \rho_{n} & 0 \\
0 & \cosh \frac{\eta}{2} \rho_{n}-\sinh \frac{\eta}{2} \rho_{n}
\end{array}\right) \phi_{0} .
$$

Now, we choose $\phi_{0}=(1,1)^{T}$ in $(34)$, then we have

$$
\phi_{n}=\left(\begin{array}{c}
e^{\eta \rho_{n} / 2} \\
e^{-\eta \rho_{n} / 2}
\end{array}\right)
$$

Substitute (35) into (22), then, by (28), we obtain the new solutions of the cmKdV-SG equations (13):

$$
u^{\prime}(x, t)=n \Pi+4 \tan ^{-1}\left(e^{\eta \rho_{n}}\right), \quad n=0, \pm 1, \pm 2, \pm 3, \ldots
$$

Consequently, the solution of (11) is

$$
\begin{gathered}
u^{\prime}(r, \theta)=n \Pi+4 \tan ^{-1}\left(e^{\eta \rho_{n}}\right), \\
\rho_{n}=(24)^{1 / 4} \frac{r}{h}-(24)^{-1 / 4} \frac{h \theta}{\beta} k, \\
k=\eta^{2}-\frac{\alpha}{\eta^{2}}(-1)^{n} \quad n=0, \pm 1, \pm 2, \pm 3, \ldots
\end{gathered}
$$

By means of the same procedures above, we obtain the solution of $\mathrm{mKdV}$ equation:

(i) when $\alpha=0$ in (13), we obtain the $\mathrm{mKdV}$ equation in $u_{x}$

$$
\left(u_{x}\right)_{t}+\left(u_{x}\right)_{x x x}+\frac{3}{2}\left(u_{x}\right)^{2}\left(u_{x}\right)_{x}=0
$$

and its solutions is

$$
u^{\prime}(x, t)=4 \frac{\partial}{\partial x} \tan ^{-1}\left(e^{\eta \rho}\right), \quad \rho=x-\eta^{2} t
$$

(ii) when $\alpha=0$ in (11), we obtain the $\mathrm{mKdV}$ equation in $u_{r}$

$$
\beta\left(u_{r}\right)_{\theta}+\frac{1}{16} h^{4}\left(u_{r}\right)^{2}\left(u_{r}\right)_{r}+\frac{1}{24} h^{4}\left(u_{r}\right)_{r r r}=0,
$$

and its solutions is

$$
\begin{gathered}
u^{\prime}(r, \theta)=4 \frac{\partial}{\partial r} \tan ^{-1}\left(e^{\eta \rho}\right), \\
\rho=(24)^{1 / 4} \frac{r}{h}-(24)^{-1 / 4} \frac{h \theta}{\beta} \eta^{2} .
\end{gathered}
$$


Now, we use a known traveling wave solutions for the cmKdV-SG equations to generate a new solution for the $\mathrm{cmKdV}-\mathrm{SG}$ equations by means of the BTs.

We will find a new traveling wave solutions $u^{\prime}(x, t)$ of the cmKdV-SG equations (13) and substitute these solution into the corresponding matrices $P$ and $Q$. Next we solve (22) for $\phi_{1}$ and $\phi_{2}$. Then by (22) and the corresponding BTs (28) we will obtain the new solution classes. We take

$$
u=4 \tan ^{-1}\left(e^{\eta \rho}\right), \quad \rho=x-k t, \quad k=\eta^{2}-\frac{\alpha}{\eta^{2}},
$$

as a traveling wave solution class of the $\mathrm{cmKdV}$-SG equations (13). The traveling wave known solution of the cmKdV-SG equations takes the form

$$
u=u(\rho), \quad \rho=x-k t .
$$

In this case the AKNS system (5) and (6) has a general solution. Let us consider the more general case. Suppose that the components $q$ and $r$ of the matrix $P$ are function of $\rho$ $[8,12]$ :

$$
q=q(\rho), \quad r=r(\rho)
$$

then the components $A, B$ and $C$ of the matrix $Q$ as determined by (6) are also functions of $\rho$ :

$$
A=A(\rho), \quad B=B(\rho), \quad C=C(\rho) .
$$

Under these assumptions, the following result holds, which is crucial in the subsequent exact solution. The quantity

$$
\beta_{1}=\left(A+k \frac{\eta}{2}\right)^{2}+(B+k q)(C+k r)
$$

is constant with respect to $\rho$ (or $x$ and $t$ ). Using the result of [13] and the constant $\beta_{1}$ defined by (46) is greater than zero and therefore the corresponding solution of the AKNS system (5) and (6) is:

$$
\left[\begin{array}{l}
\phi_{1} \\
\phi_{2}
\end{array}\right]=\left[\begin{array}{c}
c_{1}(C+k r)^{-1 / 2}\left[\left(A+k \frac{\eta}{2}\right) \sinh \omega\left(\xi+c_{2}\right)+\omega \cosh \omega\left(\xi+c_{2}\right)\right] \\
c_{1}(C+k r)^{1 / 2} \sinh \omega\left(\xi+c_{2}\right)
\end{array}\right], \quad \text { when } \beta_{1}>0, \omega^{2}=\beta_{1}
$$

where $c_{1}$ and $c_{2}$ are constants and

$$
\xi=t+\int \frac{r d \rho}{C+k r} \text {. }
$$

Now applying the results obtained here and the known traveling wave solutions for the cmKdV-SG equations respectively to construct new solution class of the corresponding cmKdV-SG equations by means of the BTs. The constant $\beta_{1}$ and $\xi$ defined by (46), (48) can be determined by using (42)

$$
\begin{aligned}
\xi= & t-\left[\frac{\eta^{2}}{2 \eta^{4}+2 \alpha}-\frac{8 \eta^{10}}{9 \eta^{12}+3 \eta^{8} \alpha-5 \alpha^{2} \eta^{4}+\alpha^{3}}\right] \rho \\
& -\left(\frac{\eta}{12 \eta^{4}-4 \alpha}\right) e^{-2 \eta \rho} \\
& -\left[\frac{4 \eta^{9}}{9 \eta^{12}+3 \eta^{8} \alpha-5 \alpha^{2} \eta^{4}+\alpha^{3}}\right] \\
& \times \ln \left(e^{2 \eta \rho}+\frac{\alpha-3 \eta^{4}}{\alpha+\eta^{4}}\right) .
\end{aligned}
$$

Consequently, we obtain $\Gamma$ from (47) for $\beta_{1}>0$

$$
\Gamma=(C+k r)^{-1}\left[\left(A+k \frac{\eta}{2}\right)+\omega \operatorname{coth} \omega\left(\xi+c_{2}\right)\right],
$$

then substituting this $\Gamma$ into the BTs (28) and using (42), we arrive at the new solution $u^{\prime}$ of the cmKdV-SG equations (13) corresponding to the known traveling wave solution class (42), then

$$
u^{\prime}(x, t)=4\left[\tan ^{-1}\left(e^{\eta \rho}\right)+\tan ^{-1} \Gamma\right]
$$

Consequently, the solution of (11) is

$$
\begin{gathered}
u^{\prime}(r, \theta)=4\left[\tan ^{-1}\left(e^{\eta \rho}\right)+\tan ^{-1} \Gamma\right], \\
\rho=(24)^{1 / 4} \frac{r}{h}-(24)^{-1 / 4} \frac{h \theta}{\beta} k, \quad k=\eta^{2}-\frac{\alpha}{\eta^{2}} .
\end{gathered}
$$

By means of the same procedures above,

(i) we obtain the solution of $\mathrm{mKdV}$ equation (38),

$$
\begin{gathered}
u^{\prime}(x, t)=4 \frac{\partial}{\partial x}\left[\tan ^{-1}\left(e^{\eta \rho}\right)+\tan ^{-1} \Gamma\right], \\
\rho=x-\eta^{2} t, \\
\xi=t+\frac{7}{9 \eta^{2}} \rho-\frac{1}{12 \eta^{3}} e^{-2 \eta \rho}-\frac{4}{9 \eta^{3}} \ln \left(e^{2 \eta \rho}-3\right),
\end{gathered}
$$

(ii) we obtain the solution of $\mathrm{mKdV}$ equation (40),

$$
\begin{gathered}
u^{\prime}(r, \theta)=4 \frac{\partial}{\partial x}\left[\tan ^{-1}\left(e^{\eta \rho}\right)+\tan ^{-1} \Gamma\right] \\
\rho=(24)^{1 / 4} \frac{r}{h}-(24)^{-1 / 4} \frac{h \theta}{\beta} \eta^{2}, \\
\xi=(24)^{-1 / 4} \frac{h \theta}{\beta}+\frac{7}{9 \eta^{2}} \rho-\frac{1}{12 \eta^{3}} e^{-2 \eta \rho}-\frac{4}{9 \eta^{3}} \ln \left(e^{2 \eta \rho}-3\right) .
\end{gathered}
$$

\section{Conservation Laws for the cmKdV-SG Equations}

One of the most widely accepted definitions of integrability of PDEs requires the existence of soliton solutions, that is, 
of a special kind of traveling wave solutions that interact "elastically," without changing their shapes. The analytical construction of soliton solutions is based on the general ISM. In the formulation of Zakharov and Shabat [14], all known integrable systems supporting solitons can be realized as the integrability condition of a linear problem of the form (5). Thus, an equation (5) is kinematically integrable if it is equivalent to the curvature condition

$$
P_{x}-Q_{t}+[P, Q]=0 .
$$

As mentioned in the previous sections, Sasaki [15], Chern and Tenenblat [2], and Cavalcante and Tenenblat [16] have given a geometrical method for constructing conservation laws of equations describing pss. The formal content of this method is contained in the following theorem, which may be seen as generalizing the classical discussion on conservation laws appearing in Wadati et al. [17].

Theorem 3. Suppose that $u_{t}=F\left(u, u_{x}, \ldots, u_{x^{k}}\right)$ or more generally $F\left(x, t, u, u_{x}, \ldots, u_{x^{n} t^{m}}\right)=0$ is an NLEE describing pss. The systems

$$
\begin{aligned}
& D_{x} \phi_{1}=q r+\left(\frac{D_{x} q}{q}-\eta\right) \phi_{1}-\phi_{1}^{2}, \\
& D_{t}\left(\frac{\eta}{2}+\phi_{1}\right)=D_{x}\left(A+\frac{B}{q} \phi_{1}\right), \\
& D_{x} \phi_{2}=-q r+\left(\frac{D_{x} r}{r}+\eta\right) \phi_{2}+\phi_{2}^{2}, \\
& D_{t}\left(\frac{\eta}{2}+\phi_{2}\right)=D_{x}\left(A+\frac{C}{r} \phi_{2}\right),
\end{aligned}
$$

in which $D_{x}$ and $D_{t}$ are the total derivative operators defined by

$$
\begin{gathered}
D_{x}=\frac{\partial}{\partial x}+\sum_{k=0}^{\infty} u_{k+1} \frac{\partial}{\partial u_{k}}, \\
D_{t}=\frac{\partial}{\partial t}+\sum_{k=0}^{\infty} D_{x}^{k}(f) \frac{\partial}{\partial u_{k}},
\end{gathered}
$$

are integrable on solutions of the equation $u_{t}=F\left(u, u_{x}, \ldots\right.$, $\left.u_{x^{k}}\right)$ or generally $F\left(x, t, u, u_{x}, \ldots, u_{x^{n} t^{m}}\right)=0$ [18].

This theorem provides one with at least one $\eta$-dependent conservation law of the NLEE $u_{t}=F\left(u, u_{x}, \ldots, u_{x^{k}}\right)$ or $F\left(x, t, u, u_{x}, \ldots, u_{x^{n} t^{m}}\right)=0$, to wit, (56) and (57) or ((58) and (59)). One obtains a sequence of $\eta$-independent conservation laws by expanding $\phi_{1}$ or $\phi_{2}$ in inverse powers of $\eta[19,22]$. Moreover,

$$
\phi_{2}=\sum_{n=1}^{\infty} \phi_{2}^{(n)} \eta^{-n}
$$

and the consideration of (58) yields the recursion relation

$$
\begin{gathered}
\phi_{2}^{(1)}=-q r \\
\phi_{2}^{(n+1)}=\frac{D_{x} r}{r} \phi_{2}^{(n)}+D_{x} \phi_{2}^{(n)}+\sum_{i=1}^{n-1} \phi_{2}^{(i)} \phi_{2}^{(n-i)}, \quad n \geq 1,
\end{gathered}
$$

which in turn, by replacing into (59), yields the sequence of conservation laws of equations integrable by AKNS inverse scattering found by Wadati et al. [17]. This section ends with the example.

For (13), we consider the functions of $u(x, t)$ defined by

$$
\begin{gathered}
r=\frac{u_{x}}{2}, \quad q=-\frac{u_{x}}{2}, \\
A=\frac{1}{2}\left(-\eta^{3}-\frac{\eta u_{x}^{2}}{2}+\frac{\alpha}{\eta} \cos u\right), \\
B=\frac{1}{2}\left(\eta u_{x x}+u_{x x x}+\frac{u_{x}^{3}}{2}+\eta^{2} u_{x}+\frac{\alpha}{\eta} \sin u\right), \\
C=\frac{1}{2}\left(\eta u_{x x}-u_{x x x}-\frac{u_{x}^{3}}{2}-\eta^{2} u_{x}+\frac{\alpha}{\eta} \sin u\right) .
\end{gathered}
$$

Equation (58) becomes

$$
D_{x} \phi_{2}=\frac{1}{4} u_{x}^{2}+\left(\frac{u_{x x}}{u_{x}}+\eta\right) \phi_{2}+\phi_{2}^{2} .
$$

Assume that $\phi_{2}$ can be expanded in a series of the form (61).

Equation (64) implies that $\phi_{2}$ is determined by the recursion relation

$$
\begin{gathered}
\phi_{2}^{(1)}=\frac{1}{4} u_{x}^{2}, \\
\phi_{2}^{(n+1)}=\frac{u_{x x}}{u_{x}} \phi_{2}^{(n)}+D_{x} \phi_{2}^{(n)}+\sum_{i=1}^{n-1} \phi_{2}^{(i)} \phi_{2}^{(n-i)}, \quad n \geq 1,
\end{gathered}
$$

whenever $u(x, t)$ is a solution of the the cmKdV-SG equations. This recursion relation yields a sequence of conserved densities given by the coefficients of the series in $\eta$

$$
\frac{\eta}{2}+\sum_{n=1}^{\infty} \phi_{2}^{(n)} \eta^{-n}
$$

which one obtains from (59).

\section{Conclusions}

In this paper, I show how the geometrical properties of a pss may be applied to obtain analytical results for the $\mathrm{cmKdV}$ SG equations which describe pss. It has been shown that the implementation of certain BTs for a class of NLEE requires the solution of the underlying linear differential equation whose coefficients depend on the known solution $u(x, t)$ of the NLEE. I obtain a new traveling wave solutions for the $\mathrm{cmKdV}$-SG equations by using BTs. Next, an infinite number of conservation laws is derived for the $\mathrm{cmKdV}$-SG equations just mentioned using a theorem by Khater et al. [18] and Sayed et al. [23-25].

\section{Acknowledgment}

The author would like to thank the anonymous referees for these helpful comments. The author thinks that revising the paper according to its suggestions will be quite straightforward. 


\section{References}

[1] R. Beals, M. Rabelo, and K. Tenenblat, "Bäcklund transformations and inverse scattering solutions for some pseudospherical surface equations," Studies in Applied Mathematics, vol. 81, no. 2, pp. 125-151, 1989.

[2] S. S. Chern and K. Tenenblat, "Pseudospherical surfaces and evolution equations," Studies in Applied Mathematics, vol. 74, no. 1, pp. 55-83, 1986.

[3] M. J. Ablowitz, D. J. Kaup, A. C. Newell, and H. Segur, “The inverse scattering transform-Fourier analysis for nonlinear problems," Studies in Applied Mathematics, vol. 53, no. 4, pp. 249315, 1974.

[4] K. Chadan and P. C. Sabatier, Inverse Problems in Quantum Scattering Theory, Springer, New York, NY, USA, 1977.

[5] V. E. Zakharov and A. B. Shabat, "Integration of nonlinear equations of mathematical physics by the method of inverse scattering. II," Functional Analysis and Its Applications, vol. 13, no. 3, pp. 166-174, 1979.

[6] M. Crampin, "Solitons and SL(2,R)," Physics Letters A, vol. 66, no. 3, pp. 170-172, 1978.

[7] K. Konno and M. Wadati, "Simple derivation of Bäcklund transformation from Riccati form of inverse method," Progress of Theoretical Physics, vol. 53, no. 6, pp. 1652-1656, 1975.

[8] R. M. Miura, Bäcklund Transformations, the Inverse Scattering Method, Solitons, and Their Applications, vol. 515 of Lecture Notes in Mathematics, Springer, Berlin, Germany, 1976.

[9] A. C. Scott, F. Y. F. Chu, and D. W. McLaughlin, "The soliton: a new concept in applied science," vol. 61, pp. 1443-1483, 1973.

[10] E. G. Reyes, "On geometrically integrable equations and hierarchies of pseudo-spherical type," in The Geometrical Study of Differential Equations, vol. 285 of Contemporary Mathematics, pp. 145-155, American Mathematical Society, Providence, RI, USA, 2001.

[11] K. Tenenblat, Transformations of Manifolds and Applications to Differential Equations, vol. 93 of Pitman Monographs and Surveys in Pure and Applied Mathematics, Longman, Harlow, UK, 1998.

[12] A. H. Khater, O. H. El-Kalaawy, and D. K. Callebaut, "Bäcklund transformations and exact solutions for Alfvén solitons in a relativistic electron-positron plasma," Physica Scripta, vol. 58, no. 6, pp. 545-548, 1998.

[13] A. H. Khater, D. K. Callebaut, and S. M. Sayed, "Exact solutions for some nonlinear evolution equations which describe pseudospherical surfaces," Journal of Computational and Applied Mathematics, vol. 189, no. 1-2, pp. 387-411, 2006.

[14] V. E. Zakharov and A. B. Shabat, "Exact theory of twodimensional self-focusing and one-dimensional self-modulation of waves in nonlinear media," Journal of Experimental and Theoretical Physics, vol. 34, pp. 62-69, 1972.

[15] R. Sasaki, "Soliton equations and pseudospherical surfaces," Nuclear Physics B, vol. 154, no. 2, pp. 343-357, 1979.

[16] J. A. Cavalcante and K. Tenenblat, "Conservation laws for nonlinear evolution equations," Journal of Mathematical Physics, vol. 29, no. 4, pp. 1044-1049, 1988.

[17] M. Wadati, H. Sanuki, and K. Konno, "Relationships among inverse method, Bäcklund transformation and an infinite number of conservation laws," Progress of Theoretical Physics, vol. 53, pp. 419-436, 1975.

[18] A. H. Khater, D. K. Callebaut, and S. M. Sayed, "Conservation laws for some nonlinear evolution equations which describe pseudo-spherical surfaces," Journal of Geometry and Physics, vol. 51, no. 3, pp. 332-352, 2004.

[19] E. G. Reyes, "Pseudo-spherical surfaces and integrability of evolution equations," Journal of Differential Equations, vol. 147, no. 1, pp. 195-230, 1998.

[20] E. G. Reyes, "Conservation laws and Calapso-Guichard deformations of equations describing pseudo-spherical surfaces," Journal of Mathematical Physics, vol. 41, no. 5, pp. 2968-2989, 2000.

[21] M. Antonova and A. Biswas, "Adiabatic parameter dynamics of perturbed solitary waves," Communications in Nonlinear Science and Numerical Simulation, vol. 14, no. 3, pp. 734-748, 2009.

[22] S. Johnson and A. Biswas, "Perturbation of dispersive topological solitons," Physica Scripta, vol. 84, no. 1, Article ID 015002, 2011.

[23] S. M. Sayed, A. M. Elkholy, and G. M. Gharib, "Exact solutions and conservation laws for Ibragimov-Shabat equation which describe pseudo-spherical surface," Journal of Computational and Applied Mathematics, vol. 27, no. 3, pp. 305-318, 2008.

[24] S. M. Sayed, O. O. Elhamahmy, and G. M. Gharib, "Travelling wave solutions for the KdV-Burgers-Kuramoto and nonlinear Schrödinger equations which describe pseudospherical surfaces," Journal of Applied Mathematics, Article ID 576783, 10 pages, 2008.

[25] S. M. Sayed and G. M. Gharib, "Canonical reduction of self-dual Yang-Mills equations to Fitzhugh-Nagumo equation and exact solutions," Chaos, Solitons \& Fractals, vol. 39, no. 2, pp. 492-498, 2009.

[26] A. I. Bobenko, "Surfaces in terms of 2 by 2 matrices. Old and new integrable cases," in Harmonic Maps and Integrable Systems, Aspects of Mathematics E23, pp. 83-127, Vieweg, Braunschweig, Germany, 1994.

[27] A. S. Fokas, I. M. Gelfand, F. Finkel, and Q. M. Liu, "A formula for constructing infinitely many surfaces on Lie algebras and integrable equations," Selecta Mathematica. New Series, vol. 6, no. 4, pp. 347-375, 2000.

[28] A. S. Fokas and Q. M. Liu, "Generalized conditional symmetries and exact solutions of non-integrable equations," Theoretical and Mathematical Physics, vol. 99, no. 2, pp. 571-582, 1994.

[29] A. S. Fokas and I. M. Gelfand, "Surfaces on Lie groups, on Lie algebras, and their integrability," Communications in Mathematical Physics, vol. 177, no. 1, pp. 203-220, 1996.

[30] A. S. Fokas and I. M. Gelfand, "Integrability of linear and nonlinear evolution equations and the associated nonlinear Fourier transforms," Letters in Mathematical Physics, vol. 32, no. 3, pp. 189-210, 1994.

[31] A. S. Fokas, "A unified transform method for solving linear and certain nonlinear PDEs," Proceedings of the Royal Society of London Series A, vol. 453, no. 1962, pp. 1411-1443, 1997.

[32] A. S. Fokas, "Two-dimensional linear partial differential equations in a convex polygon," Proceedings of the Royal Society of London Series A, vol. 457, no. 2006, pp. 371-393, 2001.

[33] A. S. Fokas, "Integrable nonlinear evolution equations on the half-line," Communications in Mathematical Physics, vol. 230, no. 1, pp. 1-39, 2002.

[34] A. S. Fokas and B. Pelloni, "Integral transforms, spectral representation and the $d$-bar problem," Proceedings of the Royal Society of London Series A, vol. 456, no. 1996, pp. 805-833, 2000.

[35] A. S. Fokas and L.-Y. Sung, "Generalized Fourier transforms, their nonlinearization and the imaging of the brain," Notices of the American Mathematical Society, vol. 52, no. 10, pp. 1178-1192, 2005. 


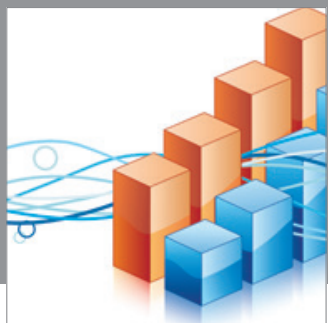

Advances in

Operations Research

mansans



The Scientific World Journal
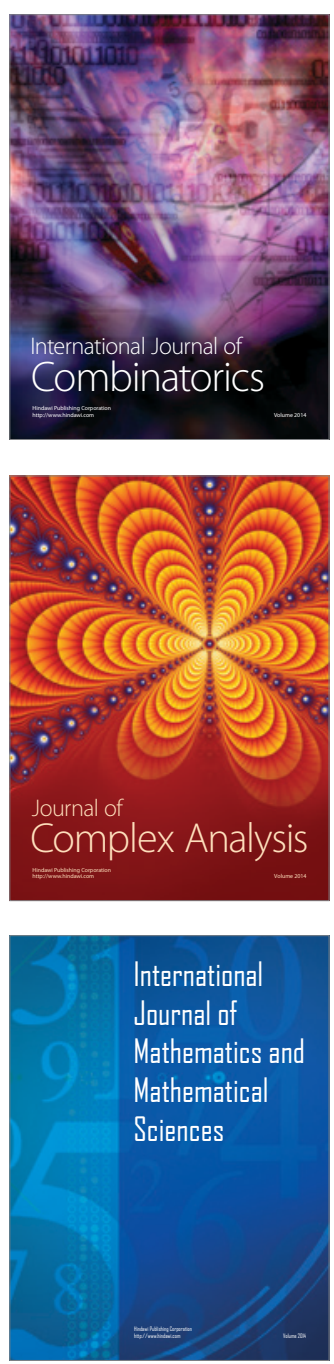
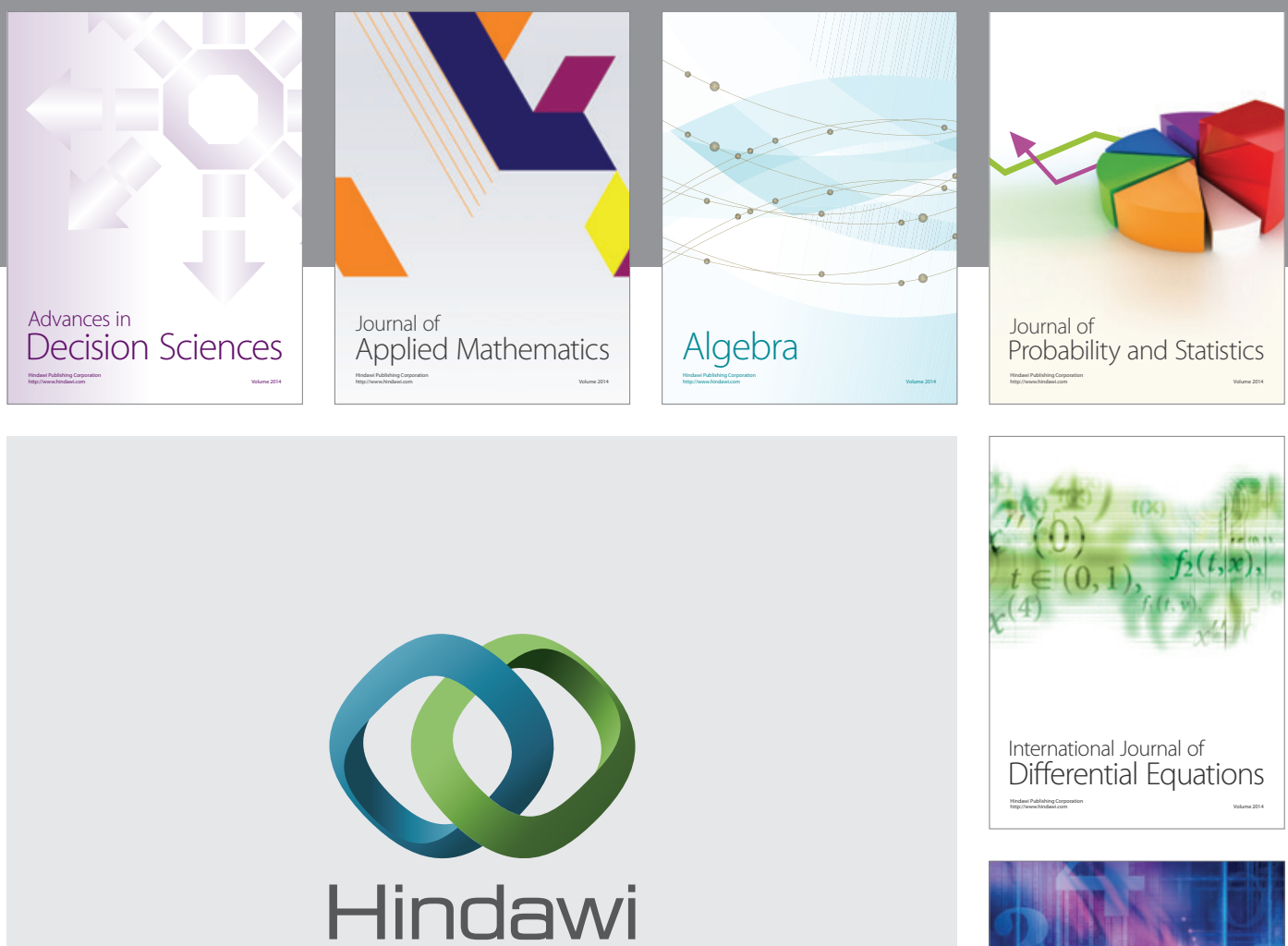

Submit your manuscripts at http://www.hindawi.com
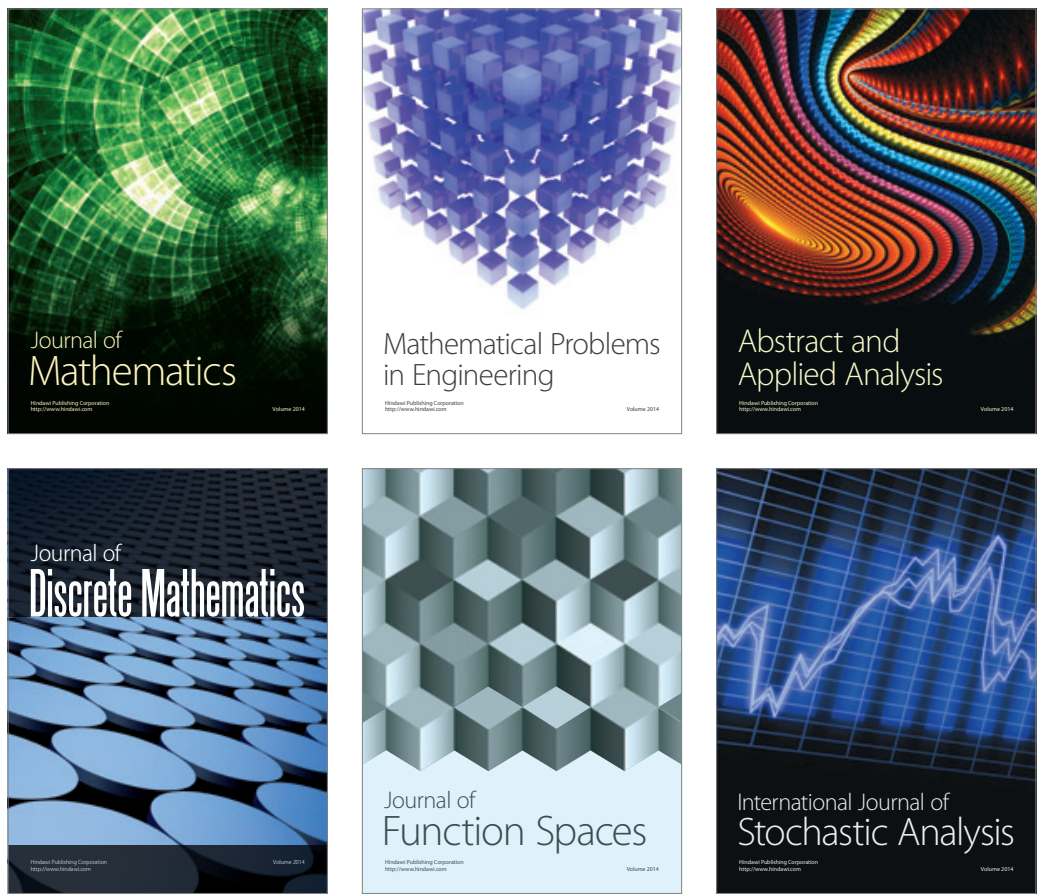

Journal of

Function Spaces

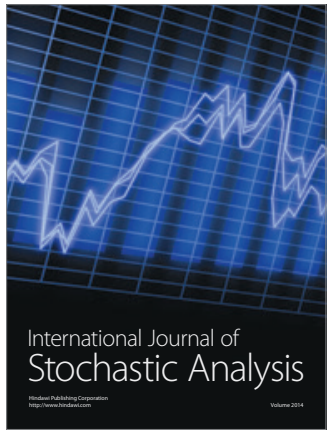

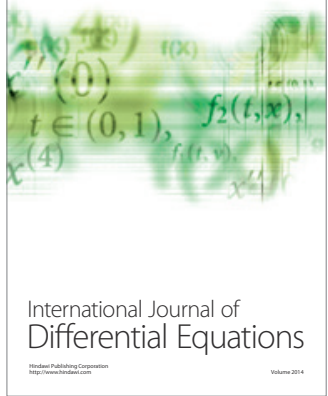
